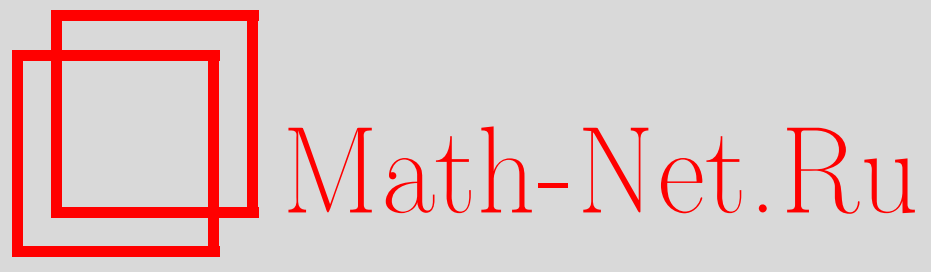

Н. И. Жукова, Концы типичных слоев полных картановых слоений, Матем. заметки, 2010, том 87, выпуск 2, 316-320

DOI: https://doi.org/10.4213/mzm6188

Использование Общероссийского математического портала Math-Net.Ru подразумевает, что вы прочитали и согласны с пользовательским соглашением http://www . mathnet.ru/rus/agreement

Параметры загрузки:

IP : 54.84 .234 .179

26 апреля 2023 г., $17: 39: 11$

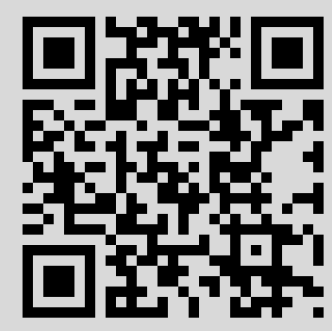




\section{Концы типичных слоев полных картановых слоений}

\section{Н. И. Жукова}

Целью данной работы является исследование топологии типичных слоев полных картановых слоений. Мы находим оценки количества концов типичных слоев, в том числе в зависимости от структурных алгебр Ли этих слоений.

1. Картановы слоения. Слоение называется картановым [1], если оно допускает в качестве трансверсальной структуры картанову геометрию. Заметим, что задание картанова слоения $(M, \mathscr{F})$ типа $\mathfrak{g} / \mathfrak{h}$ (или типа $(G, H))$ эквивалентно заданию следующих объектов:

1) группы Ли $G$ и ее замкнутой подгруппы Ли $H$ с алгебрами Ли g и $\mathfrak{h}$ соответственно;

2) главного $H$-расслоения $\pi: \mathscr{R} \rightarrow M$;

3) $H$-инвариантного слоения $(\mathscr{R}, F)$, переходящего в слоение $(M, \mathscr{F})$ при отображении $\pi$;

4) $\mathfrak{g}$-значной 1-формы $\omega$ на $\mathscr{R}$, обладающей следующими свойствами:

(i) $\omega\left(A^{*}\right)=A$ для любого $A \in \mathfrak{h}$, где $A^{*}$ - фундаментальное векторное поле, соответствующее $A$;

(ii) $R_{a}^{*} \omega=A d_{G}\left(a^{-1}\right) \omega$ для любого $a \in H$, где $A d_{G}$ - присоединенное представление группы Ли $G$ в ее алгебре Ли $\mathfrak{g}$;

(iii) для любого $u \in \mathscr{R}$ отображение $\omega_{u}: T_{u} \mathscr{R} \rightarrow \mathfrak{g}$ сюръективно, причем $\operatorname{ker} \omega=T \mathscr{F}$, где $T \mathscr{F}$ - касательное распределение к слоению $(\mathscr{R}, F)$;

(iv) производная Ли $L_{X} \omega$ равна нулю для любого векторного поля $X$, касательного к слоям слоения $(\mathscr{R}, F)$.

При этом $H$-расслоение $\pi: \mathscr{R} \rightarrow M$ называется слоеным, а слоение $(\mathscr{R}, F)$ - поднятым. Оно является трансверсально параллелизуемым или е-слоением. Далее мы рассматриваем одну компоненту связности многообразия $\mathscr{R}$, если $\mathscr{R}$ не связно.

Если группа Ли $G$ эффективно действует на однородном пространстве $G / H$, то говорят, что картаново слоение $(M, \mathscr{F})$ имеет эффективную трансверсальную картанову геометрию.

Нами показано [1], что слоение, допускающее неэффективную трансверсальную картанову геометрию, допускает также и эффективную трансверсальную картанову геометрию. Поэтому далее мы рассматриваем картановы слоения с эффективными трансверсальными картановыми геометриями. При этом наше определение [1] и определение Блюменталя [2] картанова слоения эквивалентны. Подчеркнем, что компактность слоеного многообразия $M$ не предполагается, если не оговорено противное.

ОпредЕление 1 . Картаново слоение $(M, \mathscr{F})$ называется полным, если полным является ассоциированное с ним поднятое $е$-слоение $(\mathscr{R}, F)$.

ОПРЕДЕЛЕНИЕ 2 [3]. $\mathfrak{g}$-Значная 1-форма $\omega$ называется картановой связностъю в слоеном расслоении $\pi: \mathscr{R} \rightarrow M$.

Если $\operatorname{dim} \mathscr{F}=0$, то $\omega$ - картанова связность в смысле Эресмана [4], а главное $H$-расслоение $\pi: \mathscr{R} \rightarrow M$ с картановой связностью $\omega$ есть картанова геометрия.

Работа выполнена в рамках проекта реализации ФЦП "Научные и научно-педагогические кадры инновационной России" на 2009-2011 гг. (грант № П945). 
ОПРеДЕЛЕНИЕ 3 . Говорят, что картаново слоение $(M, \mathscr{F})$ имеет нулевую трансверсалъную кривизну, если $\Omega=d \omega+(1 / 2)[\omega, \omega]=0$.

Полные картановы слоения образуют категорию $\mathfrak{C} \mathfrak{F}$, изоморфизмами в которой служат диффеоморфизмы, переводящие слои в слои, сохраняющие трансверсальную картанову геометрию.

Далее мы рассматриваем слоения $(M, \mathscr{F})$ произвольной коразмерности $q$, где $0<$ $q<n, n$ - размерность многообразия $M$.

2. Классы картановых слоений. Картановы слоения включают в себя такие широкие классы слоений, как конформные, проективные, псевдоримановы, лоренцевы, римановы, e-слоения, слоения Ли, трансверсально однородные, а также слоения с трансверсально проектируемой связностью и слоения с трансверсальной параболической структурой, где параболическая структура понимается в смысле [5].

3. Структурная алгебра Ли картанова слоения. Заметим, что слоения Ли можно определить как картановы типа $\mathfrak{g} /\{0\}$ с нулевой трансверсальной кривизной. Как известно [6], замыкание $\overline{\mathscr{L}}$ произвольного слоя $\mathscr{L}$ полного $е$-слоения $(\mathscr{R}, F)$ представляет собой гладкое подмногообразие в $\mathscr{R}$, а слоение $(\overline{\mathscr{L}}, \mathscr{F} \mid \overline{\mathscr{L}})$ является слоением Ли с всюду плотными слоями. Структурная алгебра Ли $\mathfrak{g}_{0}$ этого слоения называется структурной алгеброй Ли картанова слоения $(M, \mathscr{F})[1]$, обозначается через $\mathfrak{g}_{0}(M, \mathscr{F})$ и является инвариантом слоения $(M, \mathscr{F})$ в категории $\mathfrak{C} \mathfrak{F}$.

4. Типичные слои. Напомним, что пересечение счетного семейства открытых подмножеств в многообразии $M$ называется $G_{\delta}$-подмножеством в $M$.

Теорема 1. Пусть $(M, \mathscr{F})$ - полное картаново слоение, а $\pi: \mathscr{R} \rightarrow M-$ его слоеное расслоение. Тогда

1) сужение $\left.\pi\right|_{\mathscr{L}}: \mathscr{L} \rightarrow L:=\pi(\mathscr{L})$ проекции $\pi$ на произвольный слой $\mathscr{L}$ поднятого слоения $(\mathscr{R}, F)$ является регулярным накрывающим отображсением на слой $L$ слоения $\mathscr{F}$ с группой накрывающих преобразований, изоморфной группе голономии $\Gamma(L, x)$ слоя $L \ni x ;$

2) объединение слоев без голономии образует всюду плотное $G_{\delta}$-подмножество $M_{0}$ в $M$, дополнение которого имеет меру нуль в $M$, причем все слои из $M_{0}$ диффеоморфны между собой.

ОПРЕДЕЛЕНИЕ 4. Слои, принадлежащие $M_{0}$, называются типичными слоями картанова слоения, а многообразие, диффеоморфное слоям из $M_{0}$, называется стандартным типичным слоем этого слоения и обозначается через $L_{0}$.

5. Концы многообразий. Напомним основные понятия, связанные с множеством концов $\mathscr{E}(L)$ многообразия $L$, следуя [7]. Пусть $\mathscr{K}(L)$ - множество подмножеств $K$ из $L$, удовлетворяющих следующим условиям:

1) подмножество $K$ является связным компактным подмногообразием с краем $\partial K$ и имеет коразмерность 0 в $L$;

2) не существует предкомпактных компонент связности дополнения $L \backslash K$;

3) для любых двух различных компонент $A_{0}$ и $A_{1}$ края $\partial K$ не существует пути $h$ в $L$ такого, что $h(0) \in A_{0}, h(1) \in A_{1}$ и $h((0,1)) \cap K=\varnothing$.

Пусть $\mathscr{P}(L)$ - множество замыканий $P_{i}$ всех компонент связности дополнений $L \backslash K$, где $K$ пробегает $\mathscr{K}(L)$. Убегающей последовательностью называется убывающая последовательность $\left\{P_{n}\right\}_{n \in \mathbb{N}}$ элементов $P_{n} \in \mathscr{P}(L), P_{1} \supset \cdots \supset P_{n} \supset P_{n+1} \supset \cdots$ такая, что $\bigcap_{n} P_{n}=\varnothing$. Две убегающие последовательности $\left\{P_{n}\right\}$ и $\left\{P_{m}^{\prime}\right\}$ называются эквивалентны$\mathcal{M}$, если для каждого $n$ существует такое $m$, что $P_{m}^{\prime} \subset P_{n}$. Проверка показывает, что это 
действительно отношение эквивалентности, поэтому корректно следующее определение: класс эквивалентности $\epsilon=\left[\left\{P_{n}\right\}\right]$ называется концом многообразия $L$.

Множество всех концов многообразия $L$ обозначается через $\mathscr{E}(L)$ и наделяется топологией следующим образом. Рассмотрим $\epsilon=\left[\left\{P_{n}\right\}\right] \in \mathscr{E}(L)$ и $P \in \mathscr{P}(L)$. Выполнение включения $P_{n} \subset P$ для некоторого $n$ обозначается через $\epsilon \triangleleft P$. Пусть

$$
\mathscr{E}(P):=\{\epsilon \in \mathscr{E}(L) \mid \epsilon \triangleleft P\} .
$$

Для любого $P \in \mathscr{P}(L)$ множество $\mathscr{E}(P)$ непусто. При этом семейство

$$
\Sigma:=\{\mathscr{E}(P) \mid P \in \mathscr{P}(L)\}
$$

образует базу некоторой топологии на множестве концов $\mathscr{E}(L)$. Множество концов $\mathscr{E}(L)$, наделенное этой топологией, представляет собой компактное, вполне несвязное, хаусдорфово, метризуемое топологическое пространство.

Для многообразия $L$ существует такая компактификация $L^{*}$, что топологическое пространство концов $\mathscr{E}(L)$ гомеоморфно подпространству $L^{*} \backslash L$ в $L^{*}$. При этом компактификация $L^{*}$ характеризуется следующими свойствами [8]:

1) топологическое пространство $L^{*}$ связно;

2) подмножество $L$ открыто в $L^{*}$;

3) подпространство $L^{*} \backslash L$ вполне несвязно;

4) для любой точки $\epsilon \in L^{*} \backslash L$ и любой связной открытой окрестности $U$ точки $\epsilon$ в $L^{*}$ подмножество $U \backslash\left(L^{*} \backslash L\right)$ связно.

Будем обозначать $\operatorname{Card} \mathscr{E}(L)$ через $e(L)$. Заметим, что $\mathscr{E}(L)=\varnothing$ тогда и только тогда, когда многообразие $L$ компактно, что эквивалентно $e(L)=0$.

6. Основные теоремы. Пусть $\operatorname{dim} L=2$ и $L^{*}-$ компактификация $L$, указанная выше (см. п. 5). Конец $\epsilon \in \mathscr{E}(L)$ называется неориентируемым, если в точке $\epsilon \in L^{*}$ существует база открытых окрестностей $\left\{U_{j}\right\}$ таких, что $U_{j} \cap L$ - неориентируемые поверхности. В противном случае конец $\epsilon$ ориентируемый. Ориентируемый конец $\epsilon \in \mathscr{E}(L)$ называется непланарным, если в точке $\epsilon \in L^{*}$ существует база открытых окрестностей $\left\{U_{j}\right\}$ таких, что род каждой поверхности $U_{j} \cap L$ не равен нулю.

Применяя теорему А из [9] к слоению Ли $(\overline{\mathscr{L}}, \mathscr{F} \mid \overline{\mathscr{L}})$, благодаря теореме 1 , мы получаем первые два утверждения теоремы 2. Построенный ниже пример показывает, что последнее утверждение теоремы 2 также верно.

Теорема 2. Пусть $(M, \mathscr{F})$ - полное картаново слоение. Если его структурная алгебра Ли $\mathfrak{g}_{0}=\mathfrak{g}_{0}(M, \mathscr{F})$ отлична от нуля, то множество концов типичных слоев этого слоения либо конечно и состоит из одной или двух точек, либо является канторовым. Если, кроме того, $\operatorname{dim} \mathscr{F}=2$, то либо все типичные слои некомпактны и именот род 0; либо все типичные слои ориентируемы и имеют только непланарные концы; либо все кониы типичных слоев неориентируемы.

При $\mathfrak{g}_{0}=0$ множество концов типичных слоев этого слоения может быть любым конечным, счетным или канторовым.

ПримеР 1. Пусть $\xi$ - произвольная полная картанова геометрия с главным $H$-расслоением $p: P \rightarrow T$ над связным многообразием $T$. В качестве $L$ возьмем $S^{2} \backslash N$, сферу $S^{2}$ с выколотым конечным; замкнутым счетным или канторовым множеством точек $N$. Тогда множество концов многообразия $L$ равномощно $N$. Если $N$ - конечное множество, то топологическое пространство концов $\mathscr{E}\left(S^{2} \backslash N\right)$ дискретное. Для бесконечных множеств $N$ топология в $\mathscr{E}\left(S^{2} \backslash N\right)$ не является дискретной. Тривиальное слоение $\mathscr{F}:=\{L \times\{y\} \mid y \in T\}$ произведения $L \times T$ можно рассматривать как полное картаново слоение с трансверсальной картановой геометрией $\xi$, все слои которого типичны, а множество концов любого слоя либо произвольное конечное, либо счетное, либо канторово. 
ЗАмечание 1. Как показывают примеры, все указанные в теореме 2 возможности реализуются.

ЗАмечАниЕ 2. Фундаментальные результаты о концах $\mu$-почти каждого слоя ламинации на компактном пространстве с гармонической мерой $\mu$ получены Жисом [10].

Утверждение теоремы 3 опирается на теорему 1 и результат Хопфа [11], согласно которому множество концов любого некомпактного регулярного накрывающего пространства для компактного многообразия является либо одно или двух элементным, либо канторовым. Кроме того, применяется доказанное ранее автором утверждение о том, что если все слои полного картанова слоения компактны, то это слоение риманово, а пространство слоев - гладкий орбифолд [12].

Теорема 3. Если существует компактный слой полного картанова слоения $(M, \mathscr{F})$, то для стандартного типичного слоя $L_{0}$ либо множество кони,ов канторово, либо е $\left(L_{0}\right)$ конечно и равно 0, 1 или 2, причем $e\left(L_{0}\right)=0$ тогда и только тогда, когда $(M, \mathscr{F})$ компактное риманово слоение, а пространство слоев $M / \mathscr{F}$ - гладкий орбифолд.

Гектор, Мацумото и Майнез доказали [7], что для слоения Ли с всюду плотными слоями на компактном многообразии, структурная алгебра Ли которого разрешима, любой слой имеет ровно один или ровно два конца. Мы замечаем, что этот результат обобщается на слоения Ли с всюду плотными слоями, для которых компактность слоеного многообразия заменена требованием полноты этого слоения. Поскольку любое слоение Ли на компактном многообразии является полным римановым слоением, оно всегда полное.

Полное картаново слоение $(M, \mathscr{F})$ по определению имеет полное поднятое $e$-слоение $(\mathscr{R}, F)$, следовательно, полным является и слоение Ли $\left(\overline{\mathscr{L}},\left.\mathscr{F}\right|_{\mathscr{L}}\right)$ с всюду плотными слоями. Поэтому, применяя указанное выше наблюдение и теорему 1 , мы получаем утверждение теоремы 4 при выполнении условия 1).

Если трансверсальная кривизна полного картанова слоения $(M, \mathscr{F})$ равна нулю, то поднятое слоение $(\mathscr{R}, F)$ есть слоение Ли типа $\mathfrak{g} /\{0\}$. При этом структурная алгебра Ли $\mathfrak{g}_{0}$ слоения Ли с всюду плотными слоями $(\overline{\mathscr{L}}, \mathscr{F} \mid \overline{\mathscr{L}})$ является подалгеброй Ли алгебры $\mathfrak{g}$. Следовательно, если $\mathfrak{g}$ - разрешимая алгебра, то $\mathfrak{g}_{0}=\mathfrak{g}_{0}(M, \mathscr{F})$ также разрешима, т.е. из 2) следует 1). Таким образом, описана схема доказательства теоремы 4.

Теорема 4. Пусть $(M, \mathscr{F})$ - полное картаново слоение типа $\mathfrak{g} / \mathfrak{h}$ с ненулевой структурной алгеброй Ли. Тогда, если выполняется по крайней мере одно из следующих двух условий:

1) структурная алгебра $\mathfrak{g}_{0}(M, \mathscr{F})$ разрешима;

2) алгебра Ли g разрешима и слоение $(M, \mathscr{F})$ имеет нулевую трансверсальную кривизну;

то стандартный типичный слой этого слоения имеет ровно один или ровно два кониа.

Доказательство теоремы 5 , в отличие от теорем 2-4, использует не слоеное расслоение над полным картановым слоением $(M, \mathscr{F})$, а группоид голономии $G(\mathscr{F})$. Оно опирается на следующие результаты, полученные ранее автором [12], [13]:

1) группоид голономии $G(\mathscr{F})$ полного картанова слоения $(M, \mathscr{F})$ является хаусдорфовым многообразием, а канонические проекции $p_{j}: G(\mathscr{F}) \rightarrow M, j=1,2$, образуют локально тривиальные расслоения, стандартные слои которых диффеоморфны стандартному типичному слою $L_{0}$ слоения $(M, \mathscr{F})$;

2 ) для любого типичного слоя $L$ этого слоения прообраз $p_{j}^{-1}(L)$ диффеоморфен произведению многообразий $L \times L$. 
Теорема 5. Если $(M, \mathscr{F})$ - полное картаново слоение на односвязном компактном многообразии $M$, то е $\left(L_{0}\right)$ равно 0,1 или 2. Более того, $e\left(L_{0}\right)=0$ тогда и только тогда, когда $(M, \mathscr{F})$ - компактное риманово слоение, а $M / \mathscr{F}$ - гладкий односвязный компактный орбифолд.

СЛЕДСТВИЕ 1 (ВИНКеЛЬНКемПЕР [14]). Для риманова слоения $(M, \mathscr{F})$ на компактном односвязном римановом многообразии $е\left(L_{0}\right) \in\{0,1,2\}$.

\section{СПИСОК ЦИТИРОВАННОЙ ЛИТЕРАТУРЫ}

[1] Н. И. Жукова, Динамические системы и оптимизащия, Сборник статей. К 70-летию со дня рождения академика Дмитрия Викторовича Аносова, Тр. МИАН, 256, Наука, M., 2007, 115-147. [2] R. A. Blumenthal, Illinois J. Math., 31:2 (1987), 327-343. [3] R. A. Blumenthal, Michigan Math. J., 31:1 (1984), 55-63. [4] C. Ehresmann, Colloque de Topologie (Bruxelles, 1950), Georges Thone, Liege, 1950, 29-55. [5] A. Cap, H. Schichl, Hokkaido Math. J., 29:3 (2000), 453-505. [6] P. Molino, Riemannian Foliations, Progr. Math., 73, Birkhäuser Boston, Boston, MA, 1988. [7] G. Hector, S. Matsumoto, G. Meigniez, J. Math. Soc. Japan, 57:3 (2005), 753-779. [8] F. Raymond, Pacific J. Math., 10 (1960), 947-963. [9] J. Cantwell, L. Conlon, Comment. Math. Helv., 73:2 (1998), 306-336. [10] E. Ghys, Ann. of Math. (2), 141:2 (1995), 387-422. [11] H. Hopf, Comment. Math. Helv., 16:1 (1944), 81-100. [12] Н. И. Жукова, Изв. вузов. Матем., 1994, № 2, 78-81. [13] Н. И. Жукова, Вестн. Нижегородск. ун-та. Сер. матем., 2004, № 1, 73-87. [14] H.E. Winkelnkemper, Differential Geometry, Progr. Math., 32, Birkhäuser Boston, Boston, MA, 1983, 247-254.

\section{Н. И. Жукова}

Поступило

Нижегородский государственный университет

26.07.2008

им. Н. И. Лобачевского

E-mail: n.i.zhukova@rambler.ru

Исправленный вариант 19.03.2009 